\title{
LA-UR-11-06748
}

Approved for public release; distribution is unlimited.

\section{Title: \\ Approximating Line Losses and Apparent Power in AC Power Flow Linearizations}

Intended for:

Power Engineering Society General Meeting 2012

Carleton Coffrin

Pascal van Hentenryck

Russell Bent

\section{Los Alamos

Los Alamos National Laboratory, an affirmative action/equal opportunity employer, is operated by the Los Alamos National Security, LLC for the National Nuclear Security Administration of the U.S. Department of Energy under contract DE-AC52-06NA25396. By acceptance of this article, the publisher recognizes that the U.S. Government retains a nonexclusive, royalty-free license to publish or reproduce the published form of this contribution, or to allow others to do so, for U.S. Government purposes. Los Alamos National Laboratory requests that the publisher identify this article as work performed under the auspices of the U.S. Department of Energy. Los Alamos National Laboratory strongly supports academic freedom and a researcher's right to publish; as an institution, however, the Laboratory does not endorse the viewpoint of a publication or guarantee its technical correctness. 


\title{
Approximating Line Losses and Apparent Power in AC Power Flow Linearizations
}

\author{
Carleton Coffrin, Student Member, IEEE, Pascal Van Hentenryck, Member, IEEE, and Russell Bent
}

\begin{abstract}
The linearized DC model is widely used in optimization of power systems but few studies evaluate the accuracy and feasibility of its solutions. This paper studies the source of errors in the linearized DC model and proposes three new models to improve its accuracy. In particular, it proposes a cold-start model capturing line losses and hot-starts models for approximating apparent power and more accurate phase angles. All of the models are linear programs and can easily be used as a building block for more complex applications. Experimental results on well-known benchmarks show the significant benefits in accuracy of the new models and the high correlation of their solutions with AC solutions.
\end{abstract}

Index Terms-power flow, dc power flow, power system analysis, line losses, apparent power.

\begin{tabular}{ll} 
& \multicolumn{1}{c}{ NomENCLATURE } \\
$\left\|\widetilde{V}_{i}\right\|$ & Voltage magnitude of bus $i$, volts \\
$\theta_{i}^{\circ}$ & Phase angle of bus $i$, radians \\
$\theta_{i j}^{\circ}$ & Shorthand for $\theta_{i}^{\circ}-\theta_{j}^{\circ}$ \\
$\widetilde{Z}$ & Impedance \\
$\widetilde{Z}(i, j)$ & Impedance on a line from $i$ to $j$ \\
$x$ & Reactance \\
$r(i, j)$ & Resistance of a line from $i$ to $j$ \\
$\widetilde{Y}_{b u s}$ & The nodal admittance matrix \\
$b^{y}(i, j)$ & Susceptance from the $\widetilde{Y}_{b u s}(i, j)$ matrix \\
$g^{y}(i, j)$ & Conductance from the $\widetilde{Y}_{b u s}(i, j)$ matrix \\
$\widetilde{S}_{i j}$ & Apparent power on a line from $i$ to $j$, MVA \\
$\widetilde{L}_{i j}$ & Line Losses from $i$ to $j$, MVA \\
$p_{i}$ & Active power at bus $i$, MW \\
$q_{j}$ & Reactive power at bus $i$, MVar \\
$p_{i j}$ & Active power on a line from $i$ to $j$, MW \\
$q_{i j}$ & Reactive power on a line from $i$ to $j$, MVar \\
$\mathcal{P} \mathcal{N}$ & A power network \\
$N$ & A set of buses from a power network \\
$L$ & A set of lines from a power network
\end{tabular}

\section{INTRODUCTION}

$\mathbf{R}$ ECENT years have seen a significant increase of interest in power system optimization. Some common application domains include LMP-base market calculations [1], optimal transmission switching [2], [3], distribution configuration [4], expansion planning [5], vulnerability analysis [6], [7] and power system restoration [8], [9] to name a few. In general, power system optimization gives rise to challenging nonlinear optimization problems. However, due to the computational

C. Coffrin and P. Van Hentenryck are with the Department of Computer Science, Brown University, Providence RI 02912, USA.

R. Bent is with Los Alamos National Laboratory, Los Alamos NM 87545. difficulties associated with nonlinear optimization, the abovementioned applications typically resort to the widely accepted linearized DC model of power systems. This linearized DC model is attractive because its linear equations are easily integrated into larger models (e.g., a mixed-integer program) for a specific application domains.

Despite the pervasive use of the linearized DC model, very few applications have investigated its accuracy and how it might effect the quality and feasibility of solutions. Applications in power system optimization are usually interested in two quantities: generator dispatch and line apparent power. Generator dispatch is necessary for calculating ideal power prices, while apparent power is used for satisfying line loading constraints. Inaccuracies in either of these quantities can lead to significant economic costs or system failures. Even small errors in the price of generation can lead to millions of dollars in energy revenue when aggregated over one year [10]. Inaccuracies in apparent power computations can, in the worst case, lead to voltage collapse and blackout contingencies. There are thus considerable incentives to understand the behavior of the linearized DC model and improve its accuracy.

This paper begins with a study of linearized DC model, which confirms a number of its inaccuracies observed elsewhere (e.g., [11]). The paper then proposes three new linear programming models for power systems that address the main source of inaccuracies in the linearized DC model. In particular, it proposes a cold-start model that captures line losses, a hot-start model that approximates apparent power directly, and a hot-start model that improves phase angles. The techniques developed in each model are complementary and can be used together. Moreover, each of these models is a linear program and hence can easily be integrated in power optimization applications in the same way as the linearized DC model. The paper highlights the benefits of the three new models on the IEEE30 power system in detail and evaluates them on seven well-known benchmarks in power systems. The experimental results show that the new models significantly improve the accuracy of the linearized DC model and achieve high correlations with an $\mathrm{AC}$ model for active and apparent power.

The rest of the paper is organized as follows, Section II briefly reviews prior work on the accuracy of the linearized DC model and Section III defines the concepts of hot-start and cold-start models. Section IV gives a brief review on power system modeling and presents the linearized DC model. Section V presents a detailed study of the IEEE30 network. Section VI discusses the various sources of inaccuracies in the linearized DC model. Section VII presents the cold-start 
model capturing line losses. Section VIII introduces a hot-start model for improving the accuracy of bus phase angles. Section IX presents a hot-start model approximating apparent power. Section XI reports experimental results for these three models on well-known benchmarks. Section XII concludes the paper and discusses future research.

\section{A BRIEF REVIEW OF PRIOR WORK}

This section briefly reviews prior work on the accuracy of the linearized DC model. More details are given in appropriate sections in the paper. Despite the ubiquity of linearized DC model, there are surprisingly few studies on its accuracy. Three seminal references on this topic are [1], [11], [12]. The accuracy of the linearized DC model on random power networks and the Belgian high voltage network was studied in [12]. The paper concluded that a flat voltage ratio and high $x / r$ ratios are necessary for model accuracy. Reference [11] considered a number of linear models from the literature on very large power networks. They point out that incorporating line losses and using slack bus distribution are essential for scaling linearized DC models to large power networks. Lastly, reference [1] studied the accuracy of the linearized DC power flow relative to the AC power flow in a specific application domain, i.e., LMP calculations for security constrained OPFs. They report that the models behave similarly for a 37-bus system but differ more significantly on a larger 12,925-bus network. Overall, the accuracy of the linearized DC model is an active point of discussion, with most papers having an optimistic outlook (e.g., [12], [1]), while others are more pessimistic (e.g., [11]). The fact that ignoring reactive flows when calculating apparent power can lead to significant underestimations of line loading was mentioned in [13], although the paper does not explicitly consider linearized DC power flow accuracy.

\section{Cold-Start And Hot-STaRt Models}

The linearized DC model has a wide variety of application domains. These applications can provide additional information, that may challenge the assumptions behind the derivation of the linearized DC Model, or impose runtime constraints. For the purpose of this paper, it is useful to distinguish between hot-start and cold-start models (as defined in [11]). In hotstart models, a solved AC base-point solution is available and hence the linearized DC model has at its disposal additional information such as voltages. In cold start models, no such solved AC base-point solution is available and it becomes particularly relevant to determine if a linearized DC solution can be transformed into a AC solution. Hot-start models are often used where the network is operational and the network topology is relatively stable, e.g., in LMP-base market calculations, optimal line switching, and distribution configuration, and real-time security constrained economic dispatch. Coldstart models are used, for instance, security constrained unit commitment, power restoration after significant disruption, and long-term planning studies.

\section{Power System Modeling - A Brief Review}

For context, a brief review of the connections between the AC and linearized DC models is nessisary. a) AC Power Flow: The ground truth in this paper is the single-phase AC power model, which is widely accepted as a high-quality approximation of the steady-state behavior of real-world power flows. The single-phase AC power flow model uses Kirchhoff's current law and Ohm's law in the complex plane to define the active and reactive power injected at each power bus $n$, i.e.,

$$
\begin{aligned}
p_{n} & =\sum_{m \in N}\left\|\widetilde{V}_{n}\right\|\left\|\widetilde{V}_{m}\right\|\left(g^{y}(n, m) \cos \left(\theta_{n m}^{\circ}\right)+b^{y}(n, m) \sin \left(\theta_{n m}^{\circ}\right)\right) \\
q_{n} & =\sum_{m \in N}\left\|\widetilde{V}_{n}\right\|\left\|\widetilde{V}_{m}\right\|\left(g^{y}(n, m) \sin \left(\theta_{n m}^{\circ}\right)-b^{y}(n, m) \cos \left(\theta_{n m}^{\circ}\right)\right) .
\end{aligned}
$$

These AC power flow equations can be solved by iterative solution techniques such as the Newton-Raphson method [14], [15], [16]. Convergence of these methods is not guaranteed and, when the system is heavily loaded, the solution space is riddled with infeasible low-voltage solutions which are not usable in practice [17]. In fact, finding a solution to the AC power flow when a base-point solution is unavailable is often "maddeningly difficult" [1].

b) The Linearized DC Model: The linearized DC model is derived from the AC power flow model through a series of approximations justified by operational considerations. In particular, it is assumed that (1) the susceptance is large relative to the impedance $|b(n, m)| \gg|g(n, m)|$; (2) the phase angle difference $\theta_{n m}^{\circ}$ is small enough to ensure $\sin \left(\theta_{n m}^{\circ}\right) \approx \theta_{n m}^{\circ}$; and (3) the voltage magnitudes $\|\widetilde{V}\|$ are close to 1.0 and do not not vary significantly. Under these assumptions, the AC power flow equations reduce to,

$$
p_{n}=\sum_{m \in N}^{n \neq m} b^{y}(n, m)\left(\theta_{n}^{\circ}-\theta_{m}^{\circ}\right)
$$

From a computational standpoint, the linearized DC model is much more appealing than the AC model: It forms a system of linear equations that admit reliable algorithms. These linear equations can also be embedded into optimization frameworks for decision support in power systems [9], [1], [7], [2], [5], [8]. However, it is important to verify whether the assumptions of the linearized DC model holds for each application domain.

c) Implementation Choices: The linearized DC model is so pervasive that authors often forget to mention important implementation details. Indeed, reference [11] recently demonstrated that small changes to the model formulation may have a significant impact on its accuracy. Moreover, there are conflicting suggestions about how the $\widetilde{Y}_{\text {bus }}$ matrix is derived (e.g., using $1 / x$ or $-\Im\left(\widetilde{Z}^{-1}\right)$ ) [18], [19], [20], [21]. Our goal is to make the AC and DC power models as similar as possible and our implementation reflects this choice. In particular, we use the same susceptance value $b^{y}(n, m)$ in the AC and DC models and adopt the $\widetilde{Y}_{\text {bus }}$ calculation described and implemented in MATPOWER [22]. By necessity, AC solvers use a slack bus to ensure the flow balance in the network when the total power consumption is not known a priori (e.g., due to line losses). As a consequence, we also use a slack bus for the various DC models considered in this paper so that the AC and DC models can be accurately compared. It should be emphasized that the models proposed do not need a slack bus and the only reason to use a slack bus in these 


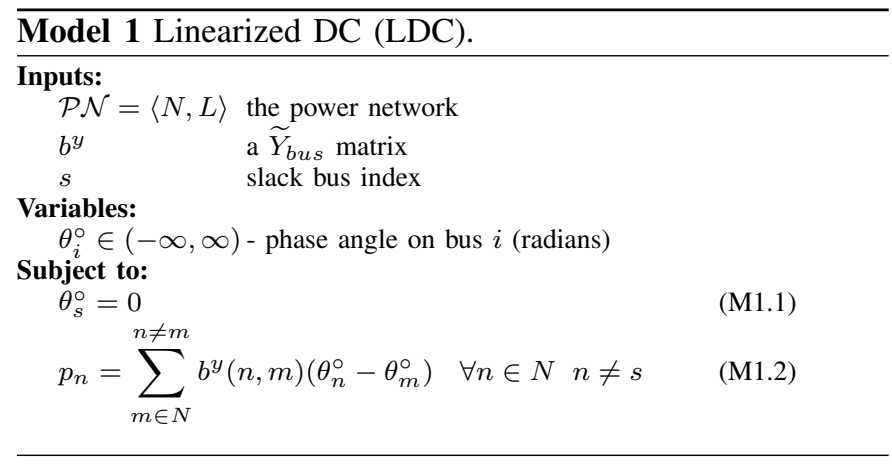

models is to allow for meaningful comparisons between the DC and AC models.

Model 1 presents the linearized DC model (LDC) used in this paper. In this paper, both the AC and DC models fix the phase angle of the slack bus to 0 . The slack bus also has no active power equation so that it can balance balance any power flow mismatch in the network. The remaining buses use Kirchhoff's current law, i.e., Equation 1, to enforce flow conservation in the power network.

\section{CAse Study: The IEEE30 Network}

To understand the accuracy of the linearized DC model, we study the IEEE30 benchmark in detail. To compare AC and DC models, we measure the same information (e.g., active power, phase angles, ...) in both models and plot their correlation. Specifically, for some measurement data (e.g., the active power of a line), the $\mathrm{x}$-axis gives the data value in the $\mathrm{AC}$ model and the $\mathrm{y}$-axis gives the data value in the DC model. As a result, the closer the plot is to the line $x=y$, the more the AC and DC models agree. We will focus on active power, bus phase angles, and apparent power. Active power and bus angles are interesting because they fully describe the solution to the linearized DC model. Apparent power is of particular interest for applications with line capacities. Obviously, in the DC model, apparent power is approximated by active power.

Figure 1 presents the correlations of active power (left), bus phase angles (center), and apparent power (right) on the IEEE30 system. The correlations are reasonably accurate, although some obvious outliers are present. The outlier in the top right corner of the active power graph (left) is due to the lack of line loss computations. The phase angle graph (center) depicts consistently larger angles in the linearized DC model, which is due in part to the assumption that $\|\widetilde{V}\|=1.0$. Indeed, in the IEEE30 network, most voltages are greater than 1.0, so the difference in phase angles must be larger in the linearized DC model to move the same amount of power. Errors in phase angles also accumulate as the power flows farther from the slack bus. The apparent power (right) is accurate in most cases but is generally underestimated by the linearized DC model, which may be problematic for applications where line loading is strongly constrained. The top-right outlier indicates a larger error due the lack of line loss calculations. The two lowerleft outliers are purely reactive flows (such as a capacitors). Ignoring reactive flow is one of the greatest weaknesses of the linearized DC model.

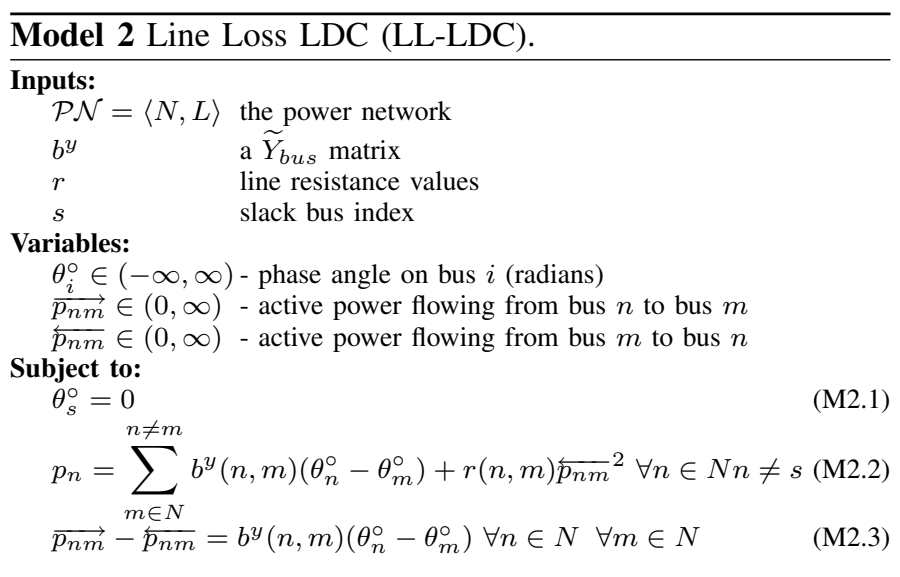

\section{INACCURACIES IN THE LINEARIZED DC MODEL}

These IEEE30 observations are consistent across several benchmarks and Section XI presents similar results on a number of well-known networks. Moreover, after extensive testing, it is possible to rank the sources of errors in decreasing order of importance as follows: (1) line losses; (2) the reactive component in apparent power; and (3) bus phase angles. Errors incurred by the sine approximation were so negligible that they can be ignored entirely. Furthermore, when the phase angle becomes large enough for the sine approximation to become significant, reactive power flow is the primary source of error and overshadows the sine approximation error. These observations are consistent with prior work (e.g., [11]) which also identifies line losses as the main source of errors in the linearized DC model.

The rest of this paper studies how to remedy some of these limitations in a linear programming setting. In other words, our goal is to obtain a linear programming model that improves the accuracy of the linearized DC model. This linear program can be reused across multiple applications. Our treatment of line losses is general and can be used for both cold-start and hot-start models. Our solutions for apparent power and phase angles are hot-start models only.

\section{APPROXIMATING LINE LOSSES}

This section derives an extension of the linearized DC model incorporating line losses, which we call the LL-LDC model (see Model 2). Consider a line from bus $n$ to $m$, its impedance $\widetilde{Z}(n, m)$, and its complex current $\widetilde{I}_{n m}$. The active and reactive line losses $\widetilde{L}_{n m}$ are given by

$$
\widetilde{L}_{n m}=\widetilde{Z}(n, m)\left\|\widetilde{I}_{n m}\right\|^{2} .
$$

The lack of an explicit representation of the line current in the linearized DC model makes it difficult to approximate this quantity. However, the derivation of the linear DC model assumes $\left|p_{n m}\right| \approx\left\|\widetilde{I}_{n m}\right\|$. Since only active power is modeled in linearized DC models, we restrict our attention to active line losses only, i.e., $\Re\left(\widetilde{L}_{n m}\right)$. The combination of the equation for active line power and the line loss calculation results in the following line loss approximation:

$$
\Re\left(\widetilde{L}_{n m}\right)=\Re(\widetilde{Z}(n, m))\left[b^{y}(n, m)\left(\theta_{n}-\theta_{m}\right)\right]^{2}
$$



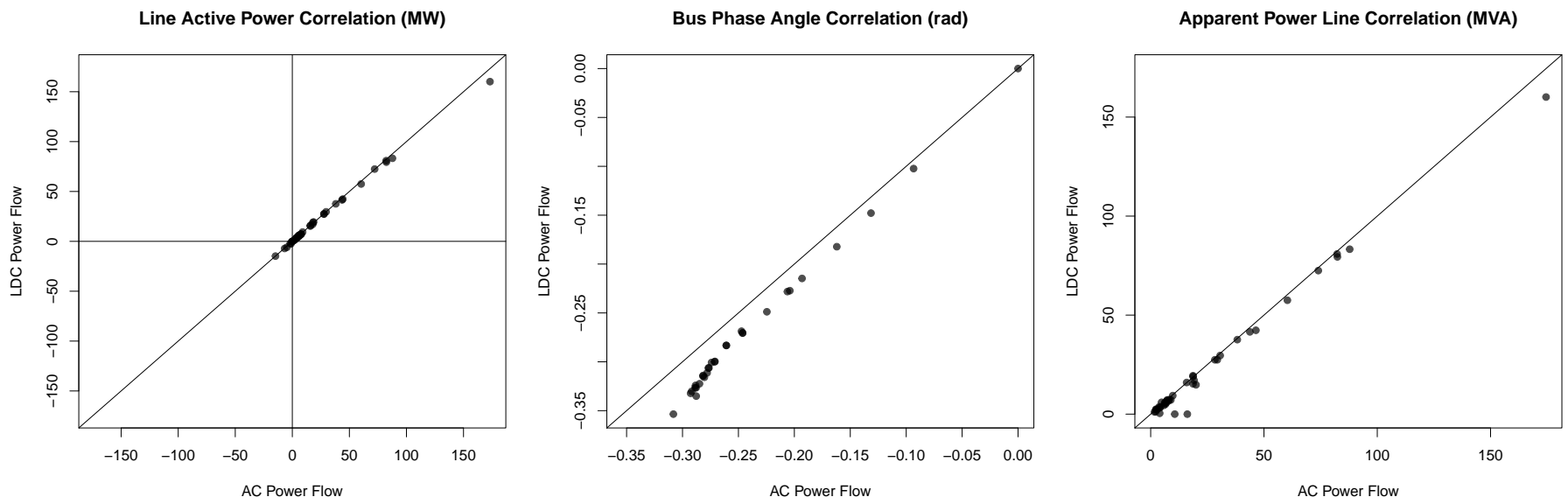

Fig. 1. Accuracy of the Linearized DC Model on Active Power (left), Bus Phase Angles (center), and Apparent Power (right).

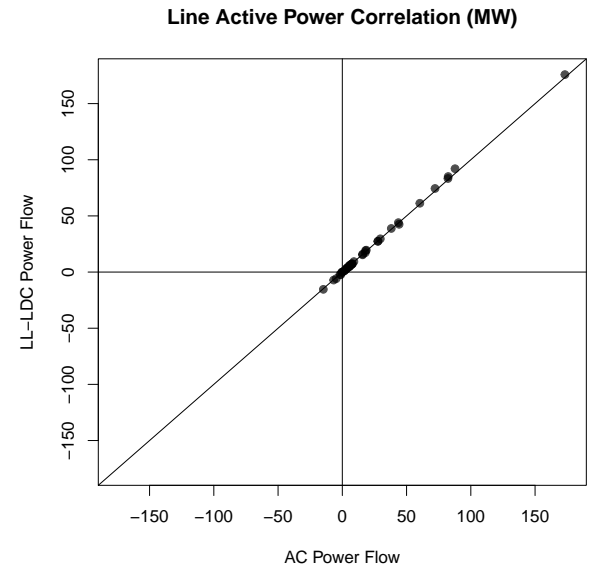

Fig. 2. Active Power in the LL-LDC Model.

To obtain a linear programming approximation of Equation 3, the first step consists in determining the flow direction on every line, since active line losses are incurred at the bus "sending" the power. Because the flow direction is not known a priori, the LL-LDC model uses two new nonnegative variables for each line, $\overrightarrow{p_{n m}}$ and $\overleftarrow{p_{n m}}$, which are linked by the constraint

$$
\overrightarrow{p_{n m}}-\overleftarrow{p_{n m}}=b^{y}(n, m)\left(\theta_{n}^{\circ}-\theta_{m}^{\circ}\right)
$$

where $\overrightarrow{p_{n m}}$ represents the flow from bus $i$ to bus $j$ and $\overleftarrow{p_{n m}}$ the flow from bus $n$ to bus $m$. The power equation for a line $(n, m)$ now becomes

$$
p_{n m}=b^{y}(n, m)\left(\theta_{n}^{\circ}-\theta_{m}^{\circ}\right)+r(n, m){\overleftarrow{p_{n m}}}^{2}
$$

since the line losses must be removed from the bus receiving the power. The second step consists in using a piecewise linear approximation of the quadratic term ${\overleftarrow{p_{n m}}}^{2}$ which is a standard technique in linear programming. In our case studies, the quadratic function is approximated in 10 pieces in the range $0 \leq \overleftarrow{p_{n m}} \leq b^{y}(n, m)$. The resulting, more accurate, definition of $p_{n m}$ can then be used in line capacity constraints.

Figure 2 evaluates the LL-LDC model on the IEEE30 benchmark. The results indicate a significant improvement in accuracy for active power. The remaining error is primarily due to the assumption that $\left|p_{n m}\right| \approx\left\|\widetilde{I}_{n m}\right\|$. Extensive results on the LL-LDC model are given in Table I to be discussed later and show consistent improvements on many benchmarks.

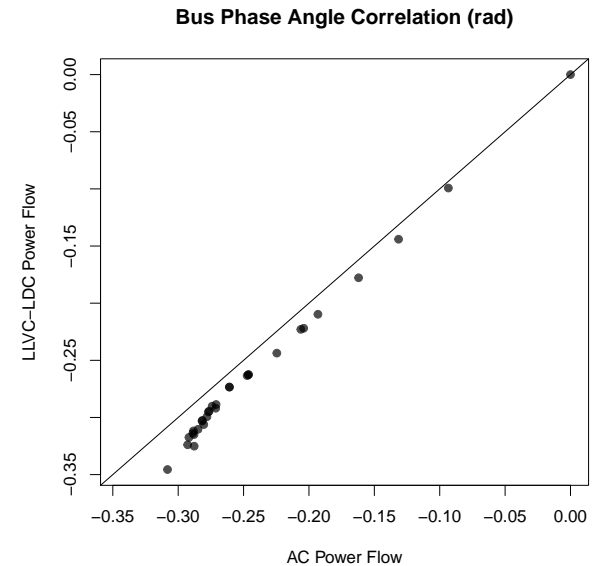

Fig. 3. Bus Phase Angles in the LLVC-LDC Model.

Other approaches were developed for incorporating line losses in the linearized DC model which are recognized as a source of significant errors [11], [1]. The most common method is to use an $\mathrm{AC}$ solver to calculate the line losses and then add those losses to the loads in the linearized DC model (e.g., [1]). However, the assignment of losses to load points is arbitrary and could remove resonable solutions form the DC power flow model. Other approaches have used an AC solution to fit a linear model of losses (e.g., [11]). This approach becomes inaccurate when line flows change significantly. Both of these approaches require a solved $\mathrm{AC}$ base point, which is not the case of our LL-LDC model. The approach presented here has similarities to the line loss modeling in [4] for a distribution line switching problem. Their derivation is quite different however: It reasons in terms of current and does not reason about the flow direction.

\section{Improving Phase Angle ACCURACy}

The linearized DC model consistently over-estimates the phase angle difference because of three factors: (1) the absence of phase angle constraints from reactive power; (2) the lack of transformer models; (3) the assumption that all $\|\widetilde{V}\|=1.0$. Reactive power and transformers are typically out of the scope of linearized DC model but, in the hot-start context, the voltage assumption can be remedied to some extent. It is sufficient to 
use the equation

$$
p_{n m}=\left\|\widetilde{V}_{n}^{h}|| \mid \widetilde{V}_{m}^{h}\right\| b^{y}(n, m)\left(\theta_{n}-\theta_{m}\right)
$$

for the power on line $(n, m)$ where $\widetilde{V}_{i}^{h}$ is the value of voltage $\widetilde{V}_{i}$ in a solved AC base-point. Figure 3 evaluates the resulting LLVC-LDC model (linearized DC model with line losses and voltage correction) with respect to the accuracy of the bus phase angles. The LLVC-LDC model produces some improvements which are consistent over several benchmarks, although there remains significant room for improvements. Note that integrating bus voltages into the power flow equations is suggested in [11] although phase angle accuracy is never explicitly discussed.

\section{Approximating Apparent Power}

Capacity constrained power flow applications, such as [1], [2], [5], [7], [8], [9], should ideally reason over apparent power to determine line loading. The linearized DC model ignores reactive power and approximates apparent power by active power. Unfortunately, this approximation can lead to significant underestimation of line loading [13]. Furthermore, the contribution of reactive power to line loading often increases under network disruptions, such as $\mathrm{N}-1$ contingencies which are often used in optimization applications [23].

One route for improving estimations of apparent power is to integrate reactive flows into the linearized DC model as in the fast decoupled load flow [24]. Another option is to approximate the nonlinear behavior of reactive flows with a mixed integer programming (MIP) formulation [4]. This is an interesting approach but it may have significant computational cost as it introduces a collection of discrete variables. Our goal is different than both of these: We want a linear-programming approximation of apparent power that can be used in a variety of optimization problems and integrated easily as part of a more complex model with minimal computational costs.

This section presents a novel approach where apparent power is modeled directly in terms of phase angles and without reference to reactive power. We start by motivating the approach and then present the derivation.

\section{A. A Motivating Study}

In benchmark IEEE30, buses 2 and 5 are voltage-controlled and are connected directly by a line. Since the voltage magnitudes $\|\widetilde{V}\|$ are known constants, the apparent power on this line can be understood completely by varying the phase angle difference between the buses. Figure 4 shows how the active, reactive, and apparent power change as the phase angle difference $\theta_{n m}^{\circ}$ is varied on line $(2,5)$. Three different scenarios are presented: On the left, the voltages are fixed at the regulated values in the system specification, $\left\|\widetilde{V}_{2}\right\|=1.045,\left\|\widetilde{V}_{5}\right\|=1.010$; In the center, the voltages are set to 1.000 to investigate the assumption of the linearized DC model; on the right, the voltages are set to the extreme values $\left\|\widetilde{V}_{2}\right\|=1.050$ and $\left\|\widetilde{V}_{5}\right\|=0.950$ to understand a worsecase scenario. The figure only depicts the graphs for angle differences up to $\pm \pi / 3$, since power systems are designed to keep phase angles small (for instance, they may remain

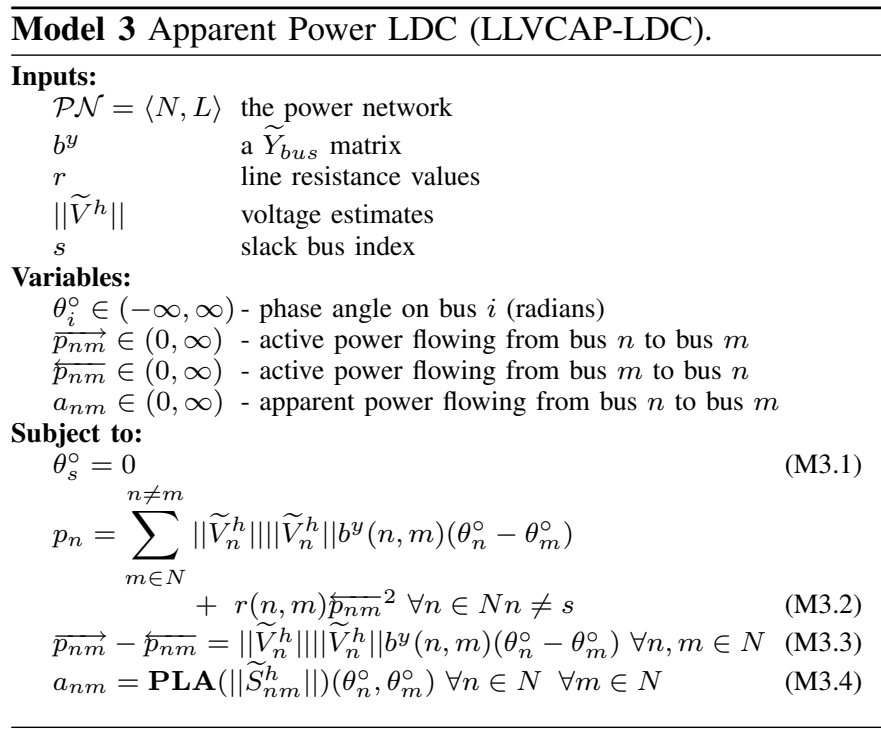

between $1^{\circ}$ and $\left.5^{\circ}[12]\right)$. Small phase angles are an operational assumption of the linearized DC model, which makes phase angles differences around $0^{\circ}$ of particular interest.

The center graph indicates that the estimation $\left\|\widetilde{S}_{n m}\right\|=$ $\left|p_{n m}\right|$ of the linearized DC model is highly accurate when the voltage magnitudes are the same. The left graph shows that the linearized DC estimation is rather accurate, except for small and large angle differences. For an angle difference of zero, there is no real power in the linearized DC model, although the apparent power never reaches zero. The right graph indicates that the linearized DC model is very inaccurate when the voltage magnitudes differ substantially. In particular, it is rather inaccurate for small angle differences, which is the common case. Such significant differences in voltage are rare in normal operations but they may arise in applications with large topological changes such as expansion planning and power restoration after significant disruptions.

Perhaps the most interesting observation is the commonalities between all of these scenarios. Despite the nonlinearity of apparent power, all of the graphs look reasonably convex ${ }^{1}$ and have a minimum at $\theta_{n m}^{\circ}=0$. This suggests that a piecewiselinear approximation of apparent power may be able to handle line capacity constraints reasonably well by using available voltages in hot-start models.

\section{B. A Piecewise Linear Approximation of Apparent Power}

We now derive a piecewise approximation of apparent power using voltage estimates. Apparent power is defined as the magnitude of the complex power $\widetilde{S}$, i.e.,

$$
\left\|\widetilde{S}_{n m}\right\|=\sqrt{p_{n m}^{2}+q_{n m}^{2}}
$$

In a hot-start context, we may assume that reasonable voltage estimates $\widetilde{V}_{n}^{h}$ are available. We can then define two functions which, given phase angles $\theta_{n}^{\circ}$ and $\theta_{m}^{\circ}$, return estimations of the active and reactive power on a line $(n, m)$ in terms of

\footnotetext{
${ }^{1}$ It is clear that this function is not truly convex by the slight curve near the edges of the domain.
} 
Line Flow in IEEE30 (in isolation)

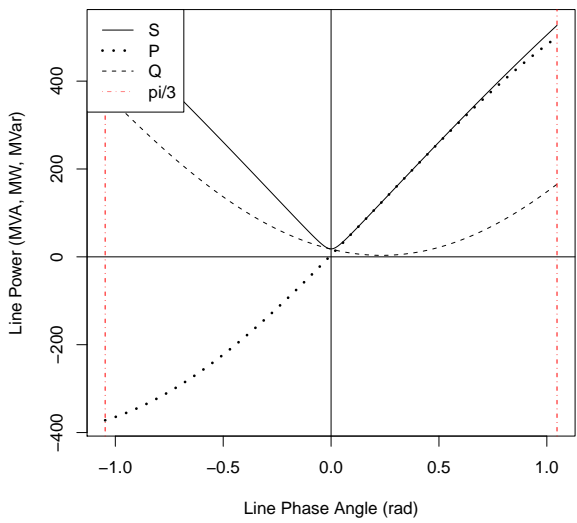

Line Flow in IEEE30 (in isolation)

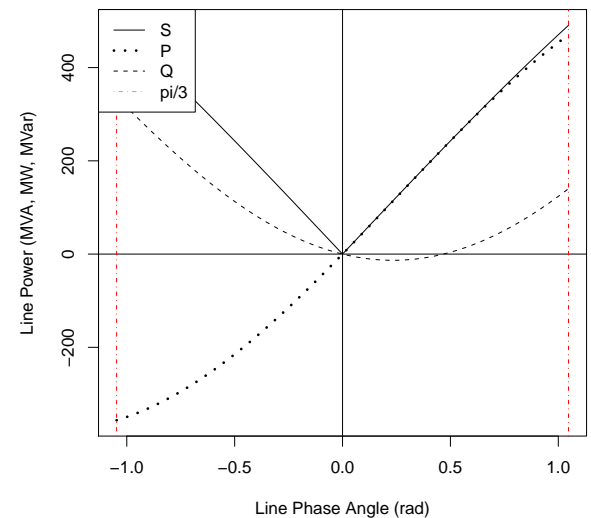

Line Flow in IEEE30 (in isolation)

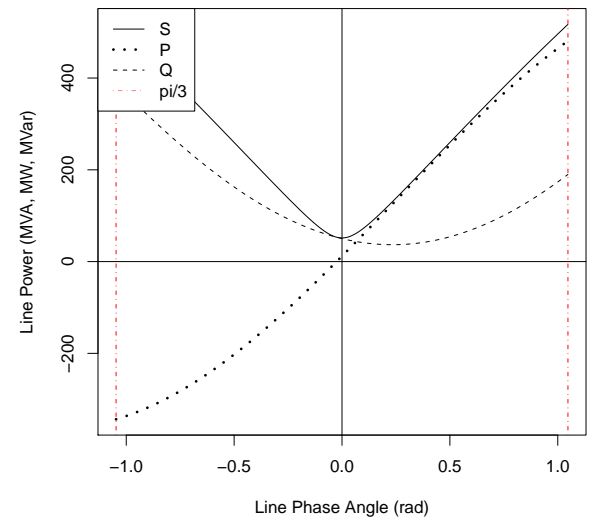

Fig. 4. Accuracy of the Linearized DC Model: Actual Voltage Magnitude (left), Magnitudes at 1.00 (center), and Magnitudes at 1.05 and 0.95 (right).

these voltages. The active power is given by the function $p_{n m}^{h}\left(\theta_{n}^{\circ}, \theta_{m}^{\circ}\right)$ defined as

$$
\begin{aligned}
& \left\|\widetilde{V}_{n}^{h}\right\|\left\|\widetilde{V}_{n}^{h}\right\| \dot{g}^{y}(n, m)+ \\
& \left\|\widetilde{V}_{n}^{h}\right\|\left\|\widetilde{V}_{m}^{h}\right\|\left(g^{y}(n, m) \cos \left(\theta_{n m}^{\circ}\right)+b^{y}(n, m) \sin \left(\theta_{n m}^{\circ}\right)\right) .
\end{aligned}
$$

where $\dot{g}^{y}(n, m)$ is the contribution of line $(n, m)$ to the diagonal values $g^{y}(n, n)$ of $\widetilde{Y}_{b u s}$. The function for reactive power $q_{n m}^{h}\left(\theta_{n}^{\circ}, \theta_{m}^{\circ}\right)$ can be defined similarly. Given phase angles $\theta_{n}^{\circ}$ and $\theta_{m}^{\circ}$, the apparent power on a line $(n, m)$ in a hot-start context can be obtained from the function

$$
\left\|\widetilde{S}_{n m}^{h}\right\|\left(\theta_{n}^{\circ}, \theta_{m}^{\circ}\right)=\sqrt{p_{n m}^{h}\left(\theta_{n}^{\circ}, \theta_{m}^{\circ}\right)^{2}+q_{n m}^{h}\left(\theta_{n}^{\circ}, \theta_{m}^{\circ}\right)^{2}}
$$

$\left\|\widetilde{S}_{n m}^{h}\right\|$ is nonlinear and cannot be used directly in a linear program. We use a piecewise linear approximation PLA $\left(\left\|\widetilde{S}_{n m}^{h}\right\|\right)$ instead and include a constraint

$$
a_{n m}=\mathbf{P L} \mathbf{A}\left(\left\|\widetilde{S}_{n m}^{h}\right\|\right)\left(\theta_{n}^{\circ}, \theta_{m}^{\circ}\right)
$$

where $a_{n m}$ denotes (an approximation of) the apparent power. $a_{n m}$ can appear in line capacity constraints, which in turn constrain the phase angles.

We call the resulting linear program (which includes line losses and voltage corrections) the LLVCAP-LDC model (Model 3). In the implementation, the piecewise linear approximation uses 13 constraints to approximate the convex hull of the apparent power, with breakpoints chosen in the range of $\pm \pi / 12$ to achieve high accuracy for small angle differences.

A key advantage of the LLVCAP-LDC model is to define apparent power in terms of the bus phase angles, which can then be integrated in many applications and allows for significant changes in power flow. This contrasts with other approaches (e.g., [13]) that suggest restating capacity constraints in terms of active power using the voltages and reactance on the line.

Figure 5 (top) revisits the correlation of apparent power using the LLVCAP-LDC model (the graph for the linearized DC model is given in Figure 1 (right)). The outliers are essentially eliminated in the LLVCAP-LDC model which generally overestimates apparent power on the lines. This is preferable in practice, since line capacities must be strictly satisfied. The remaining inaccuracies are due to differences in bus phase angles between the AC and LLVCAP-LDC models.
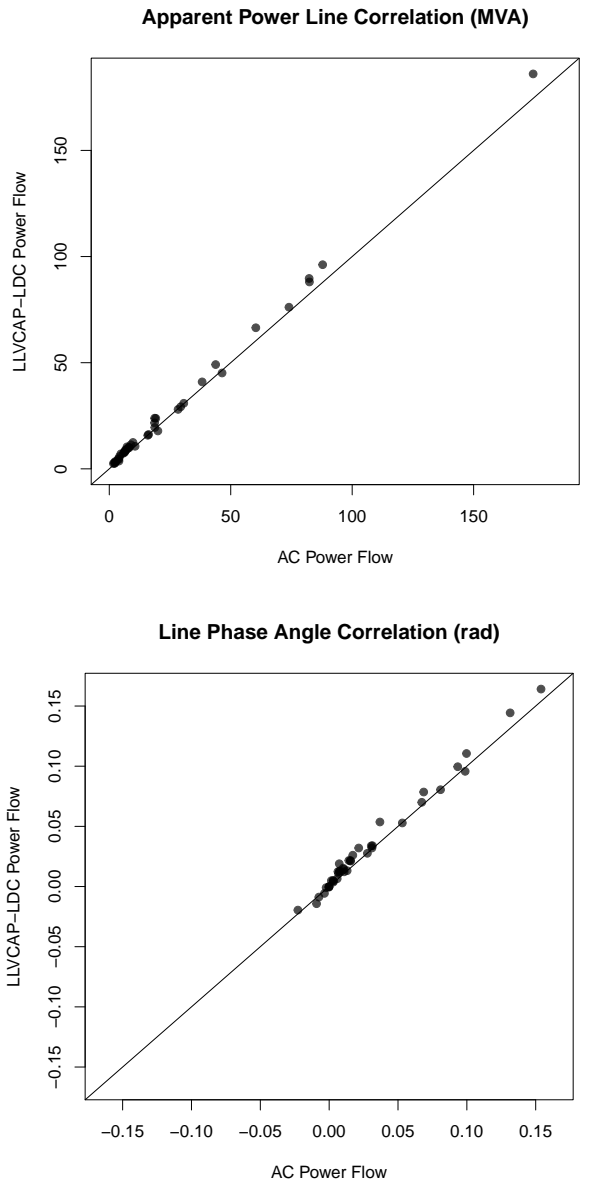

Fig. 5. Accuracy of the Apparent Power (top) and Phase Angle Differences (bottom) in the LLVCAP-DC Model.

These differences are illustrated Figure 5 (bottom). Improving the accuracy of bus phase angles would also improve the approximation of apparent power as discussed in Section VIII.

\section{$\mathrm{X}$. Computational Implications}

The linearized DC improvements proposed herein are linear programs and hence can be solved optimally in polynomial time. Moreover, they can be naturally integrated in MIP models used for many important applications such as those described in [1], [2], [3], [4], [5], [6], [7], [8], [9]. Such an integration would produce significant gains in accuracy at minimal computation costs. 
TABLE I

Active Power Flow AcCuracy Comparison

\begin{tabular}{|c|c|c|c|c|c|c|c|c|}
\hline \multirow[t]{2}{*}{ Benchmark } & \multicolumn{8}{|c|}{ Active Power (MW) } \\
\hline & Corr & $\mu(\Delta)$ & $\max (\Delta)$ & $\delta(\arg \max (\Delta))$ & $\mu(\delta)$ & $\max (\delta)$ & $\Delta(\arg \max (\delta))$ & underapproximation $(\%)$ \\
\hline ieee14 & 0.9994 & 1.392 & 10.64 & 6.783 & 6.052 & 24.33 & 0.3927 & 65 \\
\hline mp24 & 0.9989 & 5.659 & 19.7 & 23.65 & 6.447 & 29.89 & 6.656 & 47.06 \\
\hline ieee 30 & 0.9993 & 1.046 & 13.1 & 7.562 & 6.406 & 31.23 & 0.5646 & 80.49 \\
\hline mp30 & 0.9993 & 0.2964 & 2.108 & 19.36 & 3.086 & 19.36 & 2.108 & 82.93 \\
\hline ieee57 & 0.9989 & 1.494 & 8.216 & 8.054 & 105.8 & 4195 & 0.9607 & 52.56 \\
\hline ieee 118 & 0.9963 & 4.086 & 56.57 & 44.86 & 238.2 & 20350 & 2.199 & 54.19 \\
\hline ieeedd17 & 0.9972 & 4.933 & 201.3 & 13.84 & 15.2 & 215 & 0.5265 & 50.71 \\
\hline \multicolumn{9}{|c|}{ The LL-LDC Model } \\
\hline ieee14 & 0.9998 & 0.9104 & 3.875 & 5.131 & 5.214 & 22.96 & 0.3707 & 45 \\
\hline mp24 & 0.9997 & 3.685 & 14.88 & 6.67 & 4.686 & 18.93 & 4.215 & 58.82 \\
\hline ieee30 & 0.9998 & 0.7343 & 4.307 & 4.909 & 5.751 & 29.3 & 0.5298 & 56.1 \\
\hline $\mathrm{mp} 30$ & 0.9995 & 0.2399 & 1.718 & 15.77 & 2.464 & 15.77 & 1.718 & 73.17 \\
\hline ieee57 & 0.9993 & 1.008 & 4.707 & 9.797 & 108.7 & 4518 & 1.035 & 51.28 \\
\hline ieee118 & 0.9996 & 1.899 & 15.25 & 222.1 & 104.7 & 8990 & 0.9716 & 58.66 \\
\hline ieeedd17 & 0.9998 & 2.205 & 35.71 & 2.454 & 10.52 & 292.1 & 0.7154 & 55 \\
\hline
\end{tabular}

TABLE II

Phase Angle AcCuracy ACCURACy COMParison

\begin{tabular}{|c|c|c|c|c|c|c|c|c|}
\hline \multirow[t]{2}{*}{ Benchmark } & \multicolumn{8}{|c|}{ Phase Angle (rad) } \\
\hline & Corr & $\mu(\Delta)$ & $\max (\Delta)$ & $\delta(\arg \max (\Delta))$ & $\mu(\delta)$ & $\max (\delta)$ & $\Delta(\arg \max (\delta))$ & underapproximation $(\%)$ \\
\hline ieee14 & 0.9993 & 0.02487 & 0.04258 & 15.22 & 11.68 & 15.22 & 0.04258 & 100 \\
\hline mp24 & 0.9997 & 0.01334 & 0.02037 & 15.23 & 10.96 & 34.61 & 0.01274 & 4.167 \\
\hline ieee30 & 0.9981 & 0.02831 & 0.04733 & 16.45 & 11.53 & 16.45 & 0.04733 & 100 \\
\hline $\mathrm{mp} 30$ & 0.98 & 0.005658 & 0.01607 & 30.27 & 14.23 & 42.78 & 0.01598 & 86.67 \\
\hline ieee57 & 0.9898 & 0.02244 & 0.05958 & 24.1 & 10.52 & 37.94 & 0.007857 & 100 \\
\hline ieee 118 & 0.9897 & 0.0353 & 0.09208 & 91.82 & 57.59 & 2281 & 0.06523 & 4.237 \\
\hline ieeedd 17 & 0.9892 & 0.115 & 0.1395 & 16.09 & 18.76 & 36.51 & 0.1366 & 0.6173 \\
\hline \multicolumn{9}{|c|}{ The LLVC-LDC Model } \\
\hline ieee14 & 0.9994 & 0.0139 & 0.02514 & 8.984 & 6.615 & 8.984 & 0.02514 & 100 \\
\hline $\mathrm{mp} 24$ & 0.9999 & 0.00297 & 0.007173 & 3.546 & 2.286 & 7.288 & 0.002012 & 41.67 \\
\hline ieee30 & 0.9977 & 0.01972 & 0.0374 & 12.14 & 8.064 & 12.99 & 0.03737 & 100 \\
\hline mp30 & 0.9777 & 0.007322 & 0.02053 & 38.68 & 18.17 & 49.82 & 0.0186 & 90 \\
\hline ieee57 & 0.9901 & 0.02757 & 0.07275 & 21.47 & 12.95 & 35.51 & 0.007355 & 100 \\
\hline ieee 118 & 0.9989 & 0.009563 & 0.02866 & 20.1 & 7.515 & 47.09 & 0.007744 & 87.29 \\
\hline ieeedd17 & 0.9988 & 0.008753 & 0.02264 & 2.653 & 1.53 & 4.614 & 0.01726 & 17.9 \\
\hline
\end{tabular}

TABLE III

Apparent Power Flow ACCURACy COMPARISON

\begin{tabular}{|c||c||c|c|c||c|c|c||c|}
\hline Benchmark & \multicolumn{9}{c|}{ Apparent Power (MVA) } \\
& Corr & $\mu(\Delta)$ & $\max (\Delta)$ & $\delta(\arg \max (\Delta))$ & $\mu(\delta)$ & $\max (\delta)$ & $\Delta(\arg \max (\delta))$ & underapproximation $(\%)$ \\
\hline ieee14 & 0.9939 & 2.745 & 17.62 & 100 & 13.47 & 100 & 17.62 & 95 \\
\hline mp24 & 0.9911 & 8.281 & 69.48 & 44.59 & 11.86 & 56.22 & 10.79 & 67.65 \\
\hline ieee30 & 0.9952 & 2.333 & 16.18 & 100 & 17.33 & 100 & 16.18 & 87.8 \\
\hline mp30 & 0.9528 & 2.094 & 10.55 & 34.58 & 20.83 & 79.48 & 9.527 & 95.12 \\
\hline ieee57 & 0.9701 & 4.363 & 59.54 & 92.49 & 19.62 & 96.8 & 6.039 & 80.77 \\
\hline ieee118 & 0.99 & 6.332 & 90.28 & 56.49 & 18.79 & 178.2 & 14.25 & 84.92 \\
\hline ieeedd17 & 0.993 & 9.376 & 296.2 & 19.11 & 17.18 & 98.53 & 18.86 & 84.29 \\
\hline \multicolumn{8}{|c|}{ The LLVCAP-LDC Model } \\
\hline ieee14 & 0.999 & 2.68 & 9.605 & 6.071 & 14.06 & 51.25 & 0.9131 & 10 \\
\hline mp24 & 0.9995 & 3.195 & 12.9 & 10.93 & 3.715 & 12.12 & 4.921 & 17.65 \\
\hline ieee30 & 0.9988 & 2.414 & 11.53 & 6.607 & 18.29 & 47.82 & 0.8187 & 19.51 \\
\hline mp30 & 0.9838 & 2.144 & 6.322 & 25.07 & 22.3 & 58.97 & 4.21 & 21.95 \\
\hline ieee57 & 0.9958 & 3.28 & 13.54 & 10.32 & 20.35 & 76.88 & 3.274 & 25.64 \\
\hline ieee118 & 0.9983 & 4.07 & 24.4 & 15.49 & 8.752 & 45.99 & 3.678 & 20.67 \\
\hline ieeedd17 & 0.9995 & 3.001 & 41.57 & 11.86 & 5.62 & 84.35 & 1.823 & 20.36 \\
\hline
\end{tabular}

\section{Study OF STANDARD BENCHMARKS}

This section reports empirical evaluations of the LL-LDC, LLVC-LDC, and LLVCAP-LDC models on a variety of wellknown benchmarks. It reports aggregate statistics for active power (Table I), bus phase angles (Table II), and apparent power (Table III). In each table, three aggregate values are presented: Correlation (corr), absolute error $(\Delta)$, and relative error $(\delta)$. The units of the absolute error are presented in the table heading and the relative error, being a percentage, is unit-less. Both average $(\mu)$ and worst-case $(\max )$ values are presented. The worst case can often be misleading: For example a very large absolute value may actually a very small relative quantity and vice-versa. To add clarity, the tables show the relative or absolute counterpart of the value selected by the max operator using the arg max operator. The tables also include a column to indicate how often (in percentage) the measured quantity is underestimated.

Table I presents the aggregate statistics on active power in the linearized DC and LL-LDC models. The results indicate uniform improvements in active power, especially in the largest benchmarks IEEE118 and IEEEdd17. The increase in quality is staggering in IEEEdd17 where an absolute error of 
200MW in the worst case is reduced by a factor of about 6 to $35 \mathrm{MW}$. Such large errors are not uncommon for the linearized DC model on large benchmarks [11].

Table II presents the aggregate statistics on bus phase angles in the linearized DC and the LLVC-LDC models. These results show significant improvements in accuracy especially on larger benchmarks. The correlations are somewhat lower than for active power, showing room for improvements, as discussed in Section VIII and depicted in Figure 5 (bottom).

Table III presents the aggregate statistics on apparent power in the linearized DC and LLVCAP-LDC models. These results again indicate consistent and significant improvements. The improvements on the largest benchmarks are particularly significant, reducing the absolute error from about 290MVA to about 40MVA on IEEE118. Observe also that the LLVCAPLDC model generally overestimates the apparent power, which is preferable in practice where line loading capacities are strictly maintained.

\section{CONCLUSION}

Line losses, the reactive component in apparent power, and inaccurate bus phase angles are the main sources of errors in the linearized DC model. This paper proposed a number of improvements to the linearized DC model to capture line losses, improve the accuracy of phase angles, and approximate apparent power. The improvements all results in linear programming models that can be integrated in more complex applications to obtain more accurate solutions. The LL-LDC model incorporating line losses makes no assumptions and can be readily used in cold-start applications. The LLVC-LDC and LLVCAP-LDC models apply to hot-start applications, since they need voltage estimations. Each of the model produces significant improvements over the linearized DC model on a variety of well-known benchmarks.

There are many avenues for future work. First, the accuracy of phase angles should improve by integrating transformers and reactive power injection. Second, these analysis should be extended to distributed slack bus models both in the AC and DC models. Third, the enhanced models should be studied in several application domains. Finally, it would be interesting to make a detailed comparison with [13] on several applications where line capacities are critical in order to contrast the two approaches which may be orthogonal and complementary.

\section{ACKNOWLEDGMENT}

The authors would like to thank Scott Backhaus for simulating discussions about the physics of $\mathrm{AC}$ power and Brian Stott for clarifying some details of [11].

\section{REFERENCES}

[1] T. Overbye, X. Cheng, and Y. Sun, "A comparison of the ac and dc power flow models for lmp calculations," in Proceedings of the 37th Annual Hawaii International Conference on System Sciences, 2004.

[2] E. Fisher, R. O'Neill, and M. Ferris, "Optimal transmission switching," IEEE Transactions on Power Systems, vol. 23, no. 3, pp. 1346-1355, 2008.

[3] K. Hedman, R. O’Neill, E. Fisher, and S. Oren, "Optimal transmission switching with contingency analysis," IEEE Transactions on Power Systems, vol. 24, no. 3, pp. 1577-1586, aug. 2009.
[4] C. A. N. A Borghetti, M Paolone, "A Mixed Integer Linear Programming Approach to the Optimal Configuration of Electrical Distribution Networks with Embedded Generators," Proceedings of the 17th Power Systems Computation Conference (PSCC'11), Stockholm, Sweden, 2011.

[5] D. Bienstock and S. Mattia, "Using mixed-integer programming to solve power grid blackout problems," Discrete Optimization, vol. 4, no. 1, pp. 115- 141, 2007.

[6] J. Salmeron, K. Wood, and R. Baldick, "Analysis of electric grid security under terrorist threat," IEEE Transactions on Power Systems, vol. 19, no. 2, pp. 905-912, 2004.

[7] — "Worst-case interdiction analysis of large-scale electric power grids," IEEE Transactions on Power Systems, vol. 24, no. 1, pp. 96104, 2009.

[8] C. Coffrin, P. Van Hentenryck, and R. Bent, "Strategic stockpiling of power system supplies for disaster recovery." Proceedings of the 2011 IEEE Power \& Energy Society General Meetings (PES), 2011.

[9] P. Van Hentenryck, C. Coffrin, and R. Bent, "Vehicle routing for the last mile of power system restoration," Proceedings of the 17th Power Systems Computation Conference (PSCC'11), Stockholm, Sweden, 2011.

[10] D. Streiffert, R. Philbrick, and A. Ott, "A mixed integer programming solution for market clearing and reliability analysis," in Power Engineering Society General Meeting, 2005, pp. 2724- 2731 Vol. 3.

[11] B. Stott, J. Jardim, and O. Alsac, "DC Power Flow Revisited," IEEE Transactions on Power Systems, vol. 24, no. 3, pp. 1290-1300, 2009.

[12] K. Purchala, L. Meeus, D. Van Dommelen, and R. Belmans, "Usefulness of DC power flow for active power flow analysis," Power Engineering Society General Meeting, pp. 454-459, 2005.

[13] S. Grijalva, P. W. Sauer, and J. D. Weber, "Enhancement of linear ATC calculations by the incorporation of reactive power flows," IEEE Transactions on Power Systems, vol. 18, no. 2, pp. 619-624, 2003.

[14] R. Baldick, Applied Optimization: Formulation and Algorithms for Engineering Systems. Cambridge University Press, 2009.

[15] M. Crow, Computational Methods for Electric Power Systems, Second Edition (Electric Power Engineering Series). CRC Press, 2009.

[16] J. Grainger and W. S. Jr., Power System Analysis. McGraw-Hill Science/Engineering/Math, 1994.

[17] Y. Tamura, H. Mori, and S. Iwamoto, "Relationship between voltage instability and multiple load flow solutions in electric power systems,", IEEE Transactions on Power Apparatus and Systems, vol. PAS-102, no. 5, pp. 1115-1125, 1983.

[18] U. G. Knight, Power systems engineering and mathematics, by $U$. G. Knight. Pergamon Press Oxford, New York, 1972.

[19] A. J. Wood and B. F. Wollenberg, Power Generation, Operation, and Control. Wiley-Interscience, 1996.

[20] L. Powell, Power System Load Flow Analysis (Professional Engineering). McGraw-Hill Professional, 2004.

[21] A. Gomez-Exposito, A. J. Conejo, and C. Canizares, Electric Energy Systems: Analysis and Operation (Electric Power Engineering Series). CRC Press, 2008.

[22] C. Murillo-Sánchez and D. Gan, "Matpower," Ithaca, 1997.

[23] C. Coffrin, P. Van Hentenryck, and R. Bent, "Smart Load and Generation Scheduling for Power System Restoration," Proceedings of the 2012 IEEE Power \& Energy Society General Meetings (PES), 2012.

[24] B. Stott and O. Alsac, "Fast decoupled load flow," IEEE Transactions on Power Apparatus and Systems, vol. 93, no. 3, pp. 859-869, 1974.

Carleton Coffrin received a B.Sc. in Computer Science and a B.F.A. in Theatrical Design from the University of Connecticut, Storrs, CT and a M.S. from Brown University, Providence, RI. He is currently a Ph.D. candidate in the department of computer science at Brown University.

Pascal Van Hentenryck leads the Optimization Research Group at NICTA and is a professor at the University of Melbourne (on leave from Brown University). He is a fellow of the Association for the Advancement of Artificial Intelligence, the recipent of the 2002 ICS INFORMS award, the 2006 ACP Award, a honorary degree from the University of Louvain, and the Philip J. Bray award for teaching excellence. He is the author of five MIT Press books and most of this research in optimization software systems has been commercialized and is widely used in academia and industry.

Russell Bent received his $\mathrm{PhD}$ in Computer Science from Brown University in 2005 and is currently a staff scientist at LANL in the Energy and Infrastructure Analysis Group. His publications include deterministic optimization, optimization under uncertainty, infrastructure modeling and simulation, constraint programming, algorithms, and simulation. Russell has published one book and over thirty articles in peer-reviewed journals and conferences in artificial intelligence and operations research. A full list of his publications can be found at http://public.lanl.gov/rbent/. 